\title{
Avaliação de parâmetros cardiovasculares, ventilatórios e hemogasométricos de coelhos anestesiados com isofluorano ou sevofluorano e submetidos à ventilação espontânea ou controlada a volume
}

[Assessment of cardiovascular, ventilatory and blood gasometric parameters of rabbits anesthetized with isoflurane or sevoflurane and undergoing volume-controlled or spontaneous ventilation]

R.L. Carneiro ${ }^{1}$, N. Nunes ${ }^{1}$, P.C.F. Lopes $^{1}$, J.V. Moro ${ }^{1}$, R.R. Uscategui ${ }^{1}$, E.A. Belmonte ${ }^{1}$, V.F. Barbosa ${ }^{1}$, A.P. Gering ${ }^{1}$, V.J. Moraes ${ }^{2}$, E.F. Martins Filho ${ }^{2}$, D.C. Gomes Júnior ${ }^{2}$, J.M. Costa Neto ${ }^{2}$

${ }^{1}$ Universidade Estadual Paulista - Faculdade de Ciências Agrárias e Veterinárias de Jaboticabal - Jaboticabal, SP

${ }^{2}$ Universidade Federal da Bahia - Escola de Medicina Veterinária e Zootecnia - Salvador, BA

\section{RESUMO}

Avaliaram-se as ventilações mecânica controlada a volume e espontânea, por meio das variáveis hemogasométricas, cardiovasculares e ventilométricas. Distribuíram-se 28 coelhos nos grupos: GIVC (isofluorano e ventilação controlada a volume), GIVE (isofluorano e ventilação espontânea), GSVC (sevofluorano e ventilação controlada a volume) e GSVE (sevofluorano e ventilação espontânea). Induziu-se por máscara, com isofluorano (GIVE e GIVC) ou sevofluorano (GSVE e GSVC) a 1,5 CAM, em oxigênio a $100 \%$. Para manutenção anestésica, reajustou-se para 1 CAM. No GIVC e no GSVC, administrou-se rocurônio, na dose de $0,6 \mathrm{mg} / \mathrm{kg}$, seguida de infusão contínua na mesma dose de $0,6 \mathrm{mg} / \mathrm{kg} / \mathrm{h}$. No GIVE e no GSVE, foi administrado $\mathrm{NaCl}$ 0,9\% em vez de rocurônio. Iniciou-se a ventilação controlada ajustando-a de maneira a obter capnometria entre 35 e $45 \mathrm{mmHg}$. Mensuraram-se os parâmetros 60 minutos após a indução anestésica (M0), 15 minutos após o bolus de rocurônio ou $\mathrm{NaCl}$ 0,9\% (M15) e a cada 15 minutos (M30, M45 e M60). Evidenciou-se hipercapnia e acidose em GIVC, GSVC e GSVE. Concluiu-se que a ventilação mecânica controlada a volume não foi capaz de manter a normocapnia em coelhos, gerando acidose, principalmente quando se utilizou sevofluorano. $\mathrm{O}$ uso do isofluorano demonstrou maior estabilidade anestésica que o sevofluorano nesta.

Palavras-chave: coelho, anestesia inalatória, lagomorfos, respiração mecânica

\begin{abstract}
The volume-controlled mechanical ventilation and spontaneous ventilation, through haemogasometric, cardiovascular and spirometry variables were evaluated. Twenty-eight rabbits were distributed into two groups: GIVC (isoflurane and volume-controlled ventilation), GIVE (isoflurane and spontaneous ventilation), GSVC (sevoflurane and volume-controlled ventilation) and GSVE (sevoflurane and spontaneous ventilation). Induction was performed by mask with isoflurane (GIVE and GIVC) or sevoflurane (GSVE and GSVC) at 1.5 MAC in 100\% oxygen. To maintain anesthesia, MAC was reset to 1. In GIVC and GSVC groups, rocuronium was administered at a dose of $0.6 \mathrm{mg} / \mathrm{kg}$ followed by its continuous infusion $(0.6 \mathrm{mg} / \mathrm{kg} / \mathrm{h})$. In GSVE and GIVE, $0.9 \% \mathrm{NaCl}$ was administered instead of rocuronium. Controlled ventilation was started by adjusting the capnometry in order to obtain values between 35 and $45 \mathrm{mmHg}$. Parameters were measured 60 minutes after induction of anesthesia (MO), 15 minutes after the bolus of rocuronium or $0.9 \% \mathrm{NaCl}(\mathrm{M} 15)$ and every fifteen minutes (M30, M45 and M60). Hypercapnia and acidosis was evident in GIVC, GSVC and GSVE. We concluded that the volume-controlled mechanical ventilation was not able to maintain normocapnia in rabbits, producing acidosis in them, especially when using sevoflurane. The use of isoflurane showed greater stability than the sevoflurane anesthetic in the species studied.
\end{abstract}

Keywords: rabbit, inhalation anesthesia, lagomorphs, mechanical respiration

Recebido em 1 de dezembro de 2011

Aceito em 29 de novembro de 2012

E-mail: rodrigolimacarneiro27@gmail.com 


\section{INTRODUÇÃO}

A ventilação controlada a volume reside na fixação prévia do volume corrente, da frequência respiratória e da relação inspiração:expiração. $\mathrm{O}$ ventilador gera um fluxo que é interrompido ao final da inspiração; se existir a programação de uma pausa inspiratória, o fluxo cessa antes do tempo inspiratório programado. A combinação entre a ventilação controlada e o bloqueio neuromuscular possibilita a redução do consumo de oxigênio. Ademais, nessa modalidade, o uso do bloqueador se faz necessário por diminuir as interferências na mecânica respiratória autonômica durante o ato anestésico (Rotta e Steinhorn, 2007; Toufen Júnior e Carvalho, 2007).

Todos os anestésicos inalatórios deprimem a função respiratória de forma significativa. Fato este revestido de importância, no decorrer da anestesia, em que a capacidade de responder de forma adequada às alterações de $\mathrm{PaCO} 2$ e $\mathrm{PaO} 2$ encontra-se reduzida e a monitorização respiratória torna-se imprescindível. Atenção especial deve ser dada aos pacientes de risco, os quais muitas vezes já apresentam, no exame préanestésico, distúrbios de equilíbrio ácido-básico, agravamento da hipercapnia e acidose, podendo tais eventos contribuir para o óbito (Vale,1994; Fernandes e Ruiz Neto, 2002).

Flecknell et al. (1999) avaliaram os efeitos da indução anestésica com isofluorano e sevofluorano em coelhos, nos quais observaram períodos de apneia de 30 a 180 segundos durante a indução, resultando em moderada hipercapnia e acidose. Também foi observada, no ensaio experimental, significativa redução da frequência cardíaca.

O traçado eletrocardiográfico, os parâmetros ventilométricos e os gases sanguíneos sofrem interferências durante a anestesia geral, especialmente no que se refere aos anestésicos inalatórios (Martins et al., 2003). A avaliação conjunta dos parâmetros ventilométricos, hemogasométricos e eletrocardiográficos torna-se imprescindível para a análise completa das repercussões respiratórias dos fármacos inalatórios em pauta, nas condições experimentais propostas.
Este estudo tem o objetivo de comparar os efeitos das ventilações mecânica controlada a volume e espontânea, sobre as variáveis respiratórias e cardiovasculares de coelhos anestesiados com isofluorano ou sevofluorano, por meio de análises eletrocardiográfica, hemogasométrica e ventilométrica.

\section{MATERIAL E MÉTODOS}

Este experimento foi aprovado pela Comissão de Ética, Bioética e Bem-Estar Animal (CEBEA), protocolo $n^{\circ}$ 09876/54, sendo realizado de acordo com normas éticas.

Foram utilizados 28 coelhos, da raça Nova Zelândia, com peso de 2,5 a $3,5 \mathrm{~kg}$, sendo 14 machos e 14 fêmeas. Os animais foram distribuídos aleatoriamente em quatro grupos, diferenciados pelo anestésico volátil fornecido e o tipo de ventilação recebido, sendo denominados: GIVE (grupo isofluorano e ventilação espontânea); GIVC (grupo isofluorano e ventilação controlada); GSVE (grupo sevofluorano e ventilação espontânea) e GSVC (grupo sevofluorano e ventilação controlada).

A indução foi realizada por máscara naso-oral vedada, com isofluorano para GIVE e GIVC ou sevofluorano para GSVE e GSVC a 2,5 CAM, diluído em fluxo total de $1 \mathrm{~L} / \mathrm{min}$ de oxigênio a $100 \%$, fornecido por circuito anestésico com reinalação parcial de gases dotado de vaporizador calibrado (Ohmeda - modelo Isotec 5 e Sevotec 5 -Datex Ohmeda - Miami, EUA) para o agente anestésico. Obteve-se a leitura da concentração anestésica em monitor multiparamétrico (Dixtal - modelo DX 2010 ICD - Manaus, AM, Brasil) cujo sensor do analisador de gases foi adaptado à máscara durante a indução. Em seguida, os animais foram entubados com sonda de Magill de 3,0mm de diâmetro, a qual foi conectada ao aparelho de anestesia inalatória. Nesse momento, acoplou-se o sensor do analisador de gases na extremidade proximal da sonda orotraqueal conectada ao circuito anestésico, reajustando-se o vaporizador para 1,5 CAM.

Ato contínuo, efetuou-se incisão na pele na região do trígono femoral direito, para exposição da artéria femoral, na qual se introduziu um cateter para mensuração das pressões arteriais sistólica (PAS), distólica (PAD) e média (PAM) 
e coleta de sangue arterial para hemogasometria. Também foi realizada incisão na pele da região cervical lateral esquerda, com exposição da veia jugular, na qual se introduziu um cateter no interior da veia cava cranial para monitoramento da pressão venosa central (PVC), em monitor multiparamétrico.

Após o término desses procedimentos, a concentração anestésica foi reajustada para 1,0 CAM. Ato contínuo, para os animais do GIVC e GSVC, administrou-se, por via intravenosa, rocurônio na dose de $0,6 \mathrm{mg} / \mathrm{kg}$, seguido de infusão contínua na mesma dose de $0,6 \mathrm{mg} / \mathrm{kg} / \mathrm{h}$, por meio de bomba de infusão. Para os animais do GIVE e do GSVE, foi administrada solução de cloreto de sódio $(\mathrm{NaCl})$ a $0,9 \%$, seguida por infusão contínua, respeitando-se os mesmos volumes e taxas de infusão empregados no GIVC e no GSVC.

A ventilação controlada a volume foi instituída em ventilador volumétrico/pressométrico (Ohmeda - modelo 7900). Os coelhos receberam volume corrente $(\mathrm{Vt})$, com relação inspiração/expiração 1:3 e amplitude e frequência suficientes para capnometria constante entre 35 e $45 \mathrm{mmHg}$, aferida em oxicapnógrafo (Dixtal - modelo $\mathrm{CO}_{2} \mathrm{SMODX}$ 7100), não sendo mais alterada após ajuste. Os parâmetros ventilométricos foram monitorados por monitor de mecânica respiratória (Dixtal, modelo DX 8100), cujo sensor foi conectado à sonda orotraqueal, o qual forneceu: volume corrente $(\mathrm{Vt})$, volume minuto $(\mathrm{Vm})$, tempo inspiratório $\left(\mathrm{T}_{\mathrm{ins}}\right)$, pressão positiva ao final de expiração (PEEP), pressão de pico inspiratório (PIP), espaço morto das vias aéreas (Vdaw) e espaço morto alveolar (Vdalv). Aferiram-se as seguintes variáveis hemogasométricas: pressão parcial de oxigênio no sangue arterial $\left(\mathrm{PaO}_{2}\right)$, em $\mathrm{mmHg}$; pressão parcial de dióxido de carbonono no sangue arterial $\left(\mathrm{PaCO}_{2}\right)$, em $\mathrm{mmHg}$; saturação de oxi-hemoglobina no sangue arterial $\left(\mathrm{SaO}_{2}\right)$, em \%; déficit de base (DB), em $\mathrm{mEq} / \mathrm{L}$; bicarbonato $\left(\mathrm{HCO}_{3}{ }^{-}\right)$, em $\mathrm{mEq} / \mathrm{L} ; \mathrm{pH}$ do sangue arterial; e concentração de hemoglobina $\left(\mathrm{Hb}_{\mathrm{a}}\right)$, em $\mathrm{g} / \mathrm{dL}$. As variáveis foram obtidas mediante colheita de amostra de sangue, no volume de $0,3 \mathrm{~mL}$, colhida por meio do cateter inserido na artéria femoral.

O traçado eletrocardiográfico de cada animal foi avaliado observando-se a ocorrência de batimentos cardíacos de origem não sinusal. A frequência cardíaca foi obtida pela mensuração da quantidade das ondas $\mathrm{R}$ em 1 minuto em derivação DII.

Ainda foram avaliados: pressão alveolar de oxigênio $\left(\mathrm{P}_{\mathrm{A}} \mathrm{O}_{2}\right)$, diferença alvéolo-arterial de oxigênio ( $\mathrm{P}(\mathrm{A}-\mathrm{a}) \mathrm{O}_{2}$ ou $\mathrm{AaDO}_{2}$ ) e tensão de $\mathrm{CO}_{2}$ ao final da expiração $\left(\mathrm{ETCO}_{2}\right)$. A $\mathrm{P}_{\mathrm{A}} \mathrm{O}_{2}$ foi calculada usando-se a equação de gás alveolar (Bonetti e Dallan, 1997): $\mathrm{P}_{\mathrm{A}} \mathrm{O}_{2}=\left[\mathrm{FiO}_{2} \times(\mathrm{Pb}-\right.$ 47)] - $\left(\mathrm{PaCO}_{2}\right)$, em que : $\mathrm{FiO}_{2}$ é a concentração fracional de oxigênio inspirado, $\mathrm{Pb}$ é a pressão barométrica ambiente, $\mathrm{P}_{\mathrm{A}} \mathrm{O}_{2}$ é a pressão alveolar de oxigênio e $\mathrm{PaCO}_{2}$ é a pressão parcial arterial de dióxido de carbono. $\mathrm{A} \mathrm{AaDO}_{2}$ foi obtida subtraindo-se a $\mathrm{PaO}_{2}$ da $\mathrm{P}_{\mathrm{A}} \mathrm{O}_{2}$. A $\mathrm{ETCO}_{2}$ foi medida em $\mathrm{mmHg}$ e obtida por leitura direta em oxicapnógrafo (Dixtal - modelo $\mathrm{CO}_{2} \mathrm{SMODX}$ 7100).

Em todos os grupos, os parâmetros foram mensurados 60 minutos após a indução anestésica (M0), 15 minutos após o bolus de rocurônio ou $\mathrm{NaCl}$ a $0,9 \%$ (M15) e a cada 15 minutos (M30, M45 e M60). Todas as variáveis foram submetidas à análise de perfil ao nível de 5\% (Morrison, 1967; Curi, 1980).

\section{RESULTADOS E DISCUSSÃO}

Com relação às características eletrocardiográficas, as ventilações empregadas, nos grupos anestesiados com isofluorano ou sevofluorano, não produziram alterações de ritmo ou batimentos de origem não sinusal. A frequência cardíaca (FC) permaneceu dentro da faixa de normalidade para a espécie, que se encontra entre 160 e 300 bpm, segundo Fonseca et al. (1996). Dessa forma, sugere-se que a modalidade ventilatória empregada não foi capaz de interferir nesse parâmetro. Porém, o GIVC apresentou menores médias de FC, quando comparado com os demais grupos.

Os resultados deste trabalho diferem dos de Fonseca et al. (2006), que observaram taquicardia em coelhos anestesiados com propofol e isofluorano e submetidos à ventilação com pressão positiva, apesar da não significância dos resultados. A ventilação controlada a volume é uma modalidade ventilatória que exerce pressão positiva e tem como característica a diminuição do débito cardíaco, em consequência 
da diminuição do retorno venoso, devido ao aumento da pressão intratorácica, que, por sua vez, gerará aumento compensatório da frequência cardíaca. O uso de isofluorano como agente de manutenção anestésica promove diminuição da PAM, que terá como consequência aumento da FC, uma vez que o isofluorano exerce pouco efeito sobre o barorreflexo arterial.

O comportamento da frequência cardíaca no presente estudo, em especial no GIVC, é explicado pela não utilização de medicação préanestésica e fármaco indutor, que geraria redução abrupta da pressões arteriais (média, sistólica e diastólica), provocando taquicardia reflexa. As médias da pressão arterial mantiveram-se estáveis em todos os grupos avaliados.

Durante a respiração espontânea, o retorno venoso é maior na fase inspiratória, em razão da diminuição da pressão dentro do tórax, que ocasiona a migração do sangue da periferia para as grandes veias do tórax e do coração. Com respiração controlada, a pressão venosa central aumenta e o gradiente de fluxo venoso diminui, resultando em decréscimo do débito cardíaco e da pressão arterial (Jerre et al., 2007; David, 2011).

Neste trabalho, todos os grupos apresentaram médias de PAM, em quase todos os momentos, abaixo de $60 \mathrm{mmHg}$, reflexo do uso dos fármacos halogenados em estudo, os quais geram hipotensão arterial em decorrência do efeito bloqueador $\alpha_{1}$, que resulta em diminuição da resistência vascular periférica (Vale, 1994). Porém, como não houve diferenças estatísticas entre os momentos avaliados, todos os grupos mostraram-se estáveis na análise de PAM, PAS e PAD. Nos grupos anestesiados com sevofluorano, GSVC e GSVE, somente em M0 e M30, as médias de PAM foram mais altas em relação às de GIVC e GIVE.

A ventilação mecânica controlada a volume, assim como toda ventilação com pressão positiva, promove alterações cíclicas no volume sistólico do ventrículo esquerdo e do retorno venoso, gerando, consequentemente, variações na pressão arterial. Como a frequência cardíaca e o tônus vascular mantêm-se praticamente inalterados durante um único ciclo respiratório, as variações das pressões sistólica e diastólica durante a ventilação com pressão positiva ocorrem por conta das variações transitórias no volume sistólico do ventrículo esquerdo (David, 2011).

Devido aos aumentos das pressões pleural e transpulmonar na insuflação dos pulmões, os quais resultam em diminuição da pressão arterial, ocorre, de forma compensatória, recrutamento de líquidos dos vasos alveolares, que contribui para o aumento do retorno venoso e o volume sistólico do ventrículo esquerdo, gerando aumento da PAS durante a fase de inspiração (Fonseca, 2006). Esperava-se, portanto, neste estudo, que os grupos submetidos à ventilação controlada apresentassem maiores médias que os grupos submetidos à ventilação espontânea, o que não ocorreu, pois em quase todos os momentos os grupos não apresentaram diferença significativa, exceto o GSVE, que apresentou em M0 média mais alta que o GIVC.

Diante do exposto, comparando-se os modos ventilatórios deste estudo e o uso do isofluorano, apesar do não conhecimento dos valores basais, sugere-se que os achados deste trabalho não corroboram os de Lima et al. (1996), que, ao anestesiarem 10 coelhos com isofluorano, submetidos à ventilação controlada a volume, observaram diminuição da PAM nos períodos inicias do estudo.

A monitoração da PVC é indicada em intervenções em que possam ocorrer variações bruscas do volume sanguíneo (Rotta e Steinhorn, 2007), fato não ocorrido neste estudo. Portanto, a PVC manteve-se estável em todos os grupos, exceto em M15, M30 e M60, nos quais o GIVC apresentou médias superiores aos grupos submetidos à ventilação espontânea. Essas alterações pontuais são explicadas pelo fato de que, durante a respiração espontânea, a pressão nas vias aéreas diminui, enquanto na ventilação controlada ela aumenta. Com a respiração controlada, no final da fase inspiratória a PVC aumenta e o gradiente de fluxo venoso diminui (Weisman et al., 1983) (Tab. 1). 
Tabela 1. Valores médios e desvios-padrão $(\overline{\mathrm{X}} \pm \mathrm{s})$ de características cardiovasculares em coelhos anestesiados com isofluorano (GI) ou sevofluorano (GS), mantidos em ventilação espontânea (GIVE; GSVE) ou controlada a volume (GIVC; GSVC)

\begin{tabular}{|c|c|c|c|c|c|}
\hline Variável & Grupo & M0 & M15 & M30 & M45 \\
\hline $\mathrm{FC}$ & GIVE & $222 \pm 35$ & $229 \pm 35 \mathrm{~A}$ & $242 \pm 12 \mathrm{~A}$ & $229 \pm 24 \mathrm{~A}$ \\
\hline \multirow[t]{3}{*}{ (bat/min) } & GSVE & $237 \pm 9 A$ & $228 \pm 17$ & $236 \pm 15 A$ & $218 \pm 24 \mathrm{~A}$ \\
\hline & GIVC & $227 \pm 14 B$ & $208 \pm 50 B$ & $223 \pm 20 B$ & $222 \pm 23 B$ \\
\hline & GSVC & $237 \pm 23 \mathrm{~A}$ & $230 \pm 23$ & $231 \pm 20 \mathrm{~A}$ & $232 \pm 19 \mathrm{~A}$ \\
\hline PAS & GIVE & $66 \pm 5$ & $64 \pm 7$ & $57 \pm 9$ & $59 \pm 8$ \\
\hline \multirow[t]{3}{*}{$(\mathrm{mmHg})$} & GSVE & $77 \pm 13 \mathrm{~A}$ & $72 \pm 14$ & $71 \pm 13$ & $70 \pm 13$ \\
\hline & GIVC & $53 \pm 16 B$ & $66 \pm 17$ & $58 \pm 8$ & $57 \pm 8$ \\
\hline & GSVC & $71 \pm 13$ & $70 \pm 12$ & $62 \pm 13$ & $59 \pm 11$ \\
\hline PAD & GIVE & $51 \pm 7$ & $47 \pm 35 \mathrm{~A}$ & $43 \pm 3 \mathrm{~A}$ & $45 \pm 8 \mathrm{~A}$ \\
\hline \multirow[t]{3}{*}{$(\mathrm{mmHg})$} & GSVE & $61 \pm 8 A$ & $59 \pm 10$ & $54 \pm 9 \mathrm{~A}$ & $51 \pm 10 \mathrm{~A}$ \\
\hline & GIVC & $47 \pm 13 B$ & $53 \pm 10 B$ & $48 \pm 8 B$ & $45 \pm 11 B$ \\
\hline & GSVC & $62 \pm 12 \mathrm{~A}$ & $56 \pm 16$ & $53 \pm 12 \mathrm{~A}$ & $50 \pm 12 \mathrm{~A}$ \\
\hline PAM & GIVE & $57 \pm 4$ & $54 \pm 7$ & $49 \pm 4 \mathrm{~A}$ & $50 \pm 8$ \\
\hline \multirow[t]{3}{*}{$(\mathrm{mmHg})$} & GSVE & $69 \pm 10 \mathrm{~A}$ & $65 \pm 12$ & $63 \pm 8 B$ & $61 \pm 10$ \\
\hline & GIVC & $49 \pm 13 B$ & $59 \pm 12$ & $54 \pm 7$ & $53 \pm 10$ \\
\hline & GSVC & $66 \pm 12 \mathrm{~A}$ & $62 \pm 13$ & $58 \pm 13$ & $55 \pm 11$ \\
\hline PVC & GIVE & $0 \pm 2$ & $0 \pm 2$ & $1 \pm 1$ & $0 \pm 1$ \\
\hline \multirow{3}{*}{$(\mathrm{mmHg})$} & GSVE & $1 \pm 2$ & $0 \pm 3 \mathrm{~A}$ & $-1 \pm 3 \mathrm{~A}$ & $0 \pm 3$ \\
\hline & GIVC & $3 \pm 2$ & $4 \pm 2 B$ & $3 \pm 2 B$ & $3 \pm 2$ \\
\hline & GSVC & $1 \pm 4$ & $1 \pm 3$ & $2 \pm 2$ & $2 \pm 4$ \\
\hline
\end{tabular}

Médias seguidas por letras maiúsculas diferentes, na coluna, diferem entre si pela análise de perfil (P<0,05). Médias seguidas por letras minúsculas diferentes, na linha, diferem entre si pela análise de perfil $(\mathrm{P}<0,05)$.

FC: frequência cardíaca; PAS: pressão arterial sistólica; PAD: pressão arterial diastólica; PAM: pressão arterial média; PVC: pressão venosa central.

M0: mensuração 60 minutos após a indução; M15::mensuração 15 minutos após o bolus de rocurônio (GIVC, GSVC) ou NaCl 0,9\% (GIVE,GSVE); M30: mensuração 30 minutos após o bolus de rocurônio (GIVC, GSVC) ou NaCl 0,9\% (GIVE,GSVE); M45: mensuração 45 minutos após o bolus de rocurônio (GIVC, GSVC) ou NaCl 0,9\% (GIVE,GSVE); M60: mensuração 60 minutos após o bolus de rocurônio (GIVC, GSVC) ou NaCl 0,9\% (GIVE,GSVE).

A eficácia das trocas de oxigênio entre os alvéolos e os capilares pulmonares são expressas pela $\mathrm{PaO}_{2}$, que depende diretamente da $\mathrm{FiO}_{2}$, da modalidade ventilatória empregada e da relação ventilação-perfusão pulmonar (Amaral et al., 1992). Para coelhos hígidos, respirando ar ambiente, a $\mathrm{PaO}_{2}$ é estimada em $90 \mathrm{mmHg}$ (Barzago et al.,1992). Caso se obtenha $\mathrm{PaO}_{2}$ acima do valor citado, em ventilação controlada ou espontânea, isso pode ser em decorrência da alta $\mathrm{FiO}_{2}$ empregada (David, 2011). Neste estudo, em todos os grupos testados, a $\mathrm{PaO}_{2}$ apresentou valores maiores que $90 \mathrm{mmHg}$, assim tal evento pode ser atribuído à $\mathrm{FiO}_{2}=1,0$ empregada. Vale ressaltar que a ventilação controlada a volume melhora a relação ventilação-perfusão alveolar, aumentando a $\mathrm{PAO}_{2}$ e, consequentemente, a $\mathrm{PaO}_{2}$, o que facilita a difusão.

Os valores de $\mathrm{PaCO}_{2}$ considerados normais para a espécie estudada situam-se entre 20 e
46mmHg, segundo Barzago et al. (1992). Neste estudo, os grupos GIVC, GSVC e GSVE apresentaram hipercapnia. Relacionando-se tais achados aos fármacos empregados, o GSVE apresentou maiores médias de $\mathrm{PaCO}_{2}$ pelo fato de $\mathrm{o}$ anestésico produzir maior depressão respiratória que o isofluorano (Martins et al., 2003), pois, apesar de elevar a frequência respiratória, tal evento não mantém o volume minuto, aumentando, assim, a $\mathrm{PaCO}_{2}$.

Os valores de $\mathrm{ETCO}_{2}$ refletem a pressão alveolar de dióxido de carbono, sendo que a diferença entre $\mathrm{PaCO}_{2}$ e alveolar em condições normais é zero. Portanto, normalmente valores de $\mathrm{P}$ (a ET) $\mathrm{CO}_{2}$ que variam de 2 a $3 \mathrm{mmHg}$ são registrados, sendo estes indicadores do índice de espaço morto alveolar (Batista, 2011). Neste estudo, as médias de $\mathrm{P}(\mathrm{a}-\mathrm{ET}) \mathrm{CO}_{2}$, em todos os grupos, ultrapassando 2 a $3 \mathrm{mmHg}$ podem 
explicar a hipercapnia em GIVC, GSVC e GSVE.

A hipercapnia registrada em GIVC, GSVC e GSVE interferiu nas médias de $\mathrm{pH}$, que demonstraram acidose, levando em consideração que o $\mathrm{pH}$ normal para essa espécie lagomorfa situa-se entre 7,38 e 7,50 (Barzago et al., 1992). Por mecanismos de compensação, os grupos supracitados apresentaram valores de $\mathrm{HCO}_{3}^{-}$que variaram do limite superior de normalidade até acima da média para a espécie (22 a $27 \mathrm{mEq} / \mathrm{L})$, segundo Barzago et al. (1992). Os grupos submetidos à ventilação espontânea, principalmente o GIVE, que mostrou menores médias de $\mathrm{PaCO}_{2}$, apresentaram maiores médias de VT ins, VT exp e, consequentemente, de VM exp, o que reflete nas médias de $\mathrm{pH}, \mathrm{HCO}_{3}{ }^{-}$e $\mathrm{DB}$ (Johson e Morais, 2007), as quais, neste grupo, encontram-se dentro dos limites estabelecidos para a espécie. Vale ressaltar que o uso do rocurônio pode ter influenciado, sobremaneira, nas menores médias de VT nos grupos submetidos à ventilação controlada.

Fonseca (2006) não registrou diferenças nestas variáveis, quando estudou duas modalidades ventilatórias - pressão e volume controlados -, em coelhos induzidos ao choque hipovolêmico. Neste estudo, pela análise da $\mathrm{PaCO}_{2}$ e do $\mathrm{pH}$, $\mathrm{HCO}_{3}{ }^{-}$,VT ins , VT exp e VM exp, percebe-se que a ventilação controlada a volume não foi capaz de manter a normocapnia pretendida com o emprego da ventilação mecânica. Apesar do ajuste da ventilação pela $\mathrm{ETCO}_{2}$, tal evento não refletiu na $\mathrm{PaCO}_{2}$ em coelhos.

A $\mathrm{PAO}_{2}$ demonstra o balanço entre a quantidade de oxigênio fornecida pela ventilação e o quanto é transferido dos alvéolos para os capilares (Amaral et al., 1992). Portanto, uma diminuição da ventilação alveolar leva à redução da $\mathrm{PAO}_{2}$, podendo causar desequilíbrio na relação ventilação/perfusão. Neste estudo, o GIVE apresentou maiores médias de $\mathrm{PAO}_{2}$ em M15, M45 e M60; como as alterações de $\mathrm{PAO}_{2}$ são inversamente proporcionais à $\mathrm{PaCO}_{2}$ (Galas et al., 2011), sugere-se que as alterações observadas na $\mathrm{PaCO}_{2}$ foram transferidas para a $\mathrm{PAO}_{2}$, cujos menores valores da pressão parcial de dióxido de carbono foram registrados no GIVE em M15, M45 e M60. O modo ventilatório controlado a volume não se mostrou eficaz quanto à manutenção da ventilação alveolar, podendo favorecer o aparecimento de shunt arteriovenoso.

O tempo inspiratório ( $\mathrm{T}$ ins) é o período de tempo correspondente ao início até o final da inspiração. Aumentos nos valores do tempo inspiratório significam maior tempo em segundos, no qual os capilares alveolares estarão em contato com os alvéolos abertos e bem ventilados, permitindo as trocas gasosas (David, 2011). O grupos submetidos à ventilação controlada apresentaram menores médias na análise desse parâmetro e, consequentemente, maiores médias de frequência respiratória. Tal evento pode ser explicado pela determinação de uma relação I:E e volume correntes pré-fixados, no intuito de manter a $\mathrm{ETCO}_{2}$ entre 35 e $45 \mathrm{mmHg}$, o que interferiu sobremaneira na frequência respiratória, a qual, nos grupos ventilados, apresentou maiores médias, resultando em menores médias de tempo inspiratório. Tais achados contrariam os de Batista (2011) e Borges (2011), nos quais os grupos submetidos à ventilação mecânica apresentaram maiores médias de T insp.

A ventilação alveolar depende do volume corrente, do espaço morto e da frequência respiratória. A distribuição gasosa pulmonar alterada e o ventilador com seus circuitos (espaço morto mecânico) aumentam o espaço morto durante a ventilação mecânica (David , 2011).

O espaço morto anatômico (Vdaw) é representado pelas vias aéreas de condução, ou seja, da traqueia até os bronquíolos terminais, além da sonda endotraqueal e do circuito do ventilador (West, 2002). O espaço morto alveolar (Vdalv) é entendido como o volume do gás que entra nos alvéolos não submetidos à perfusão durante a respiração, ou seja, o alvéolo é ventilado, mas não é perfundido, portanto não ocorrem trocas gasosas nesses alvéolos devido a razões fisiológicas e não anatômicas (West, 2002). $\mathrm{A} \mathrm{PaCO}_{2}$ é inversamente proporcional à ventilação alveolar, que é diretamente proporcional à $\mathrm{PAO}_{2}$. $\mathrm{O}$ espaço morto fisiológico normal é menor e igual a 0,40 (David, 2011). Neste estudo, na maioria dos momentos, o GSVE apresentou médias mais altas de $\mathrm{PaCO}_{2}$ e mais baixas de $\mathrm{PAO}_{2}$, primeiramente em razão da maior depressão respiratória conferida pelo sevofluorano e também por estar em ventilação 
espontânea que favoreceu a hipercapnia, logo apresentou tendência à menor ventilação alveolar e, consequentemente, em decorrência desses aspectos, maiores médias de $\mathrm{Vdaw}$ e $\mathrm{Vd}$ alv (Tab. 2 e 3).

Tabela 2. Valores médios e desvios-padrão $(\overline{\mathrm{X}} \pm \mathrm{s})$ de características hemogasométricas em coelhos anestesiados com isofluorano (GI) ou sevofluorano (GS), mantidos em ventilação espontânea (GIVE; GSVE) ou controlada a volume (GIVC; GSVC)

\begin{tabular}{|c|c|c|c|c|c|c|}
\hline $\begin{array}{l}\text { Variáve } \\
1\end{array}$ & Grupos & M0 & M15 & M30 & M45 & M60 \\
\hline \multirow{4}{*}{$\mathrm{pH}$} & GIVE & $7,385 \pm 0,076$ & $7,394 \pm 0,073$ & $7,395 \pm 0,061 \mathrm{~A}$ & $7,392 \pm 0,055 \mathrm{~A}$ & $7,394 \pm 0,051 \mathrm{~A}$ \\
\hline & GSVE & $7,293 \pm 0,089$ & $7,287 \pm 0,141$ & $7,288 \pm 0,150$ & $7,292 \pm 0,138$ & $7,231 \pm 0,127 \mathrm{~B}$ \\
\hline & GIVC & $7,299 \pm 0,070$ & $7,314 \pm 0,096$ & $7,294 \pm 0,098$ & $7,277 \pm 0,082$ & $7,282 \pm 0,071 \mathrm{~B}$ \\
\hline & GSVC & $7,287 \pm 0,014$ & $7,269 \pm 0,023$ & $7,226 \pm 0,009 \mathrm{~B}$ & $7,225 \pm 0,033 \mathrm{~B}$ & $7,246 \pm 0,034 \mathrm{~B}$ \\
\hline \multirow{4}{*}{$\mathrm{PaO} 2$} & GIVE & $327,6 \pm 69,7$ & $350,8 \pm 50,4$ & $356,6 \pm 57,1$ & $346,2 \pm 56,5$ & $351,8 \pm 48,0$ \\
\hline & GSVE & $268,8 \pm 95,9$ & $271,1 \pm 90,9$ & $268,5 \pm 97,4$ & $273,7 \pm 96,9$ & $280,6 \pm 93,6$ \\
\hline & GIVC & $253,5 \pm 59,5$ & $239,2 \pm 75,5$ & $244,7 \pm 77,9$ & $278,9 \pm 53,5$ & $304,8 \pm 46,9$ \\
\hline & GSVC & $267,5 \pm 58,0$ & $254,6 \pm 76,3$ & $271,7 \pm 66,4$ & $275,4 \pm 60,4$ & $389,9 \pm 60,8$ \\
\hline \multirow{4}{*}{$\mathrm{PaCO} 2$} & GIVE & $42,5 \pm 7,7$ & $39,7 \pm 7,2 \mathrm{~A}$ & $40,1 \pm 6,7$ & $38,0 \pm 6,5 \mathrm{~A}$ & $37,3 \pm 6,4 \mathrm{~B}$ \\
\hline & GSVE & $58,7 \pm 27,4$ & $64,6 \pm 26,6 \mathrm{~B}$ & $61,1 \pm 27,8$ & $64,4 \pm 26,4 \mathrm{~B}$ & $73,6 \pm 24,8 \mathrm{~A}$ \\
\hline & GIVC & $53,7 \pm 5,8$ & $54,2 \pm 8,4$ & $53,4 \pm 8,3$ & $60,6 \pm 9,3 \mathrm{~B}$ & $60,2 \pm 8,3 \mathrm{~A}$ \\
\hline & GSVC & $55,9 \pm 2,1$ & $57,1 \pm 3,3$ & $58,7 \pm 2,8$ & $61,8 \pm 3,0 \mathrm{~B}$ & $64,3 \pm 3,4 \mathrm{~A}$ \\
\hline \multirow{4}{*}{$\mathrm{SO} 2$} & GIVE & $49,8 \pm 49,8$ & $83,1 \pm 37,2$ & $66,5 \pm 47,0$ & $49,9 \pm 49,9$ & $66,5 \pm 47,0$ \\
\hline & GSVE & $77,5 \pm 38,4$ & $96,5 \pm 7,2$ & $81,7 \pm 25,1$ & $93,5 \pm 9,8$ & $96,7 \pm 6,9$ \\
\hline & GIVC & $77,5 \pm 38,4$ & $58,2 \pm 58,7$ & $58,2 \pm 58,8$ & & $77,5 \pm 38,3$ \\
\hline & GSVC & $58,2 \pm 58,8$ & & & $58,2 \pm 58,8$ & $58,3 \pm 58,9$ \\
\hline \multirow{4}{*}{$\mathrm{Hb}$} & GIVE & $12,12 \pm 1,94$ & $12,35 \pm 1,34$ & $12,65 \pm 1,69$ & $11,87 \pm 1,68$ & $11,50 \pm 2,22$ \\
\hline & GSVE & $12,16 \pm 1,87$ & $12,12 \pm 1,11$ & $11,87 \pm 1,65$ & $12,18 \pm 0,80$ & $11,93 \pm 0,55$ \\
\hline & GIVC & $10,78 \pm 1,29$ & $11,60 \pm 2,80$ & $13,14 \pm 2,18$ & $12,44 \pm 1,97$ & $11,66 \pm 1,58$ \\
\hline & GSVC & $11,62 \pm 1,14$ & $10,85 \pm 1,05$ & $12,31 \pm 2,25$ & $9,57 \pm 1,68$ & $10,63 \pm 1,30$ \\
\hline \multirow{4}{*}{$\mathrm{HCO} 3$} & GIVE & $25,0 \pm 1,8$ & $23,9 \pm 1,1 \mathrm{~A}$ & $24,3 \pm 1,7$ & $22,8 \pm 1,6 \mathrm{~A}$ & $22,5 \pm 2,3 \mathrm{~A}$ \\
\hline & GSVE & $24,0 \pm 2,5$ & $28,0 \pm 1,8 \mathrm{~B}$ & $26,4 \pm 3,1$ & $28,0 \pm 1,7 \mathrm{~B}$ & $28,0 \pm 2,0 \mathrm{~B}$ \\
\hline & GIVC & $26,8 \pm 2,7$ & $26,9 \pm 2,5 \mathrm{~B}$ & $26,6 \pm 3,6$ & $27,7 \pm 2,2 \mathrm{~B}$ & $28,0 \pm 2,3 \mathrm{~B}$ \\
\hline & GSVC & $26,8 \pm 1,4$ & $26,8 \pm 1,7 \mathrm{~B}$ & $26,0 \pm 1,9$ & $24,9 \pm 2,8 \mathrm{C}$ & $26,0 \pm 2,3 \mathrm{~B}$ \\
\hline \multirow{4}{*}{ DB } & GIVE & $-0,1 \pm 2,4$ & $-0,9 \pm 1,9$ & $-0,5 \pm 1,9$ & $-1,8 \pm 1,1$ & $-2,0 \pm 2,0$ \\
\hline & GSVE & $-2,8 \pm 3,5$ & $-0,8 \pm 2,9$ & $-1,0 \pm 3,3$ & $-0,7 \pm 3,3$ & $-0,5 \pm 1,6$ \\
\hline & GIVC & $-0,8 \pm 4,2$ & $-0,9 \pm 3,5$ & $-1,3 \pm 4,3$ & $-0,9 \pm 3,1$ & $-0,5 \pm 3,2$ \\
\hline & GSVC & $-1,5 \pm 0,9$ & $-2,2 \pm 1,0$ & $-3,0 \pm 1,7$ & $-3,9 \pm 1,9$ & $-2,1 \pm 1,4$ \\
\hline
\end{tabular}

Médias seguidas por letras maiúsculas diferentes, na coluna, diferem entre si pela análise de perfil (P<0,05). Médias seguidas por letras minúsculas diferentes, na linha, diferem entre si pela análise de perfil $(\mathrm{P}<0,05)$.

pH: potencial hidrogeniônico; $\mathrm{PaO} 2$ : pressão parcial de oxigênio no sangue arterial; PaCO2: pressão parcial de dióxido de carbono no sangue arterial; SO2: saturação de oxi-hemoglobina no sangue arterial; Hb: hemoglobina; HCO3: bicarbonato; DB: déficit de base.

M0: mensuração 60 minutos após a indução; M15: mensuração 15 minutos após o bolus de rocurônio (GIVC, GSVC) ou NaCl 0,9\% (GIVE,GSVE); M30: mensuração 30 minutos após o bolus de rocurônio (GIVC, GSVC) ou NaCl 0,9\% (GIVE,GSVE); M45: mensuração 45 minutos após o bolus de rocurônio (GIVC, GSVC) ou NaCl 0,9\% (GIVE,GSVE); M60: mensuração 60 minutos após o bolus de rocurônio (GIVC, GSVC) ou NaCl 0,9\% (GIVE,GSVE). 
Tabela 3. Valores médios e desvios-padrão $(\overline{\mathrm{X}} \pm \mathrm{s})$ de características ventilométricas em coelhos anestesiados com isofluorano (GI) ou sevofluorano (GS), mantidos em ventilação espontânea (GIVE; GSVE) ou controlada a volume (GIVC; GSVC)

\begin{tabular}{|c|c|c|c|c|c|c|}
\hline Variável & Grupos & M0 & M15 & M30 & M45 & M60 \\
\hline \multirow{4}{*}{$\mathrm{SpO} 2$} & GIVE & $100 \pm 0$ & $100 \pm 0$ & $99 \pm 1$ & $100 \pm 0$ & $100 \pm 0$ \\
\hline & GSVE & $99 \pm 1$ & $99 \pm 1$ & $99 \pm 1$ & $99 \pm 1$ & $99 \pm 1$ \\
\hline & GIVC & $100 \pm 0$ & $100 \pm 0$ & $99 \pm 0$ & $99 \pm 1$ & $99 \pm 0$ \\
\hline & GSVC & $83 \pm 37$ & $83 \pm 37$ & $83 \pm 37$ & $83 \pm 37$ & $83 \pm 37$ \\
\hline \multirow{4}{*}{ ETCO2 } & GIVE & $34 \pm 5$ & $31 \pm 2$ & $30 \pm 3^{\mathrm{A}}$ & $30 \pm 3$ & $29 \pm 4$ \\
\hline & GSVE & $39 \pm 10$ & $37 \pm 13$ & $32 \pm 6$ & $38 \pm 10$ & $37 \pm 19$ \\
\hline & GIVC & $39 \pm 6$ & $32 \pm 11$ & $36 \pm 8$ & $37 \pm 7$ & $32 \pm 10$ \\
\hline & GSVC & $37 \pm 8$ & $39 \pm 5$ & $39 \pm 7 \mathrm{~B}$ & $40 \pm 5$ & $41 \pm 7$ \\
\hline \multirow{4}{*}{ FR } & GIVE & $54 \pm 11 \mathrm{~A}$ & $61 \pm 12 \mathrm{~A}$ & $66 \pm 19 A$ & $60 \pm 14 \mathrm{~A}$ & $55 \pm 10 \mathrm{~A}$ \\
\hline & GSVE & $37 \pm 10 \mathrm{~A}$ & $41 \pm 11 \mathrm{~A}$ & $40 \pm 9 \mathrm{~A}$ & $40 \pm 13 \mathrm{~A}$ & $46 \pm 11 \mathrm{~A}$ \\
\hline & GIVC & $53 \pm 7 \mathrm{~B}$ & $53 \pm 7 \mathrm{~B}$ & $53 \pm 7 \mathrm{~B}$ & $53 \pm 7 \mathrm{~B}$ & $53 \pm 7 \mathrm{~B}$ \\
\hline & GSVC & $56 \pm 7 \mathrm{~B}$ & $56 \pm 7 \mathrm{~B}$ & $58 \pm 9 \mathrm{~B}$ & $56 \pm 7 \mathrm{~B}$ & $56 \pm 7 \mathrm{~B}$ \\
\hline \multirow{4}{*}{ VT ins } & GIVE & $24,6 \pm 2,2 \mathrm{~A}$ & $24,6 \pm 2,4 \mathrm{~A}$ & $24,1 \pm 3,1 \mathrm{~A}$ & $25,3 \pm 1,9 \mathrm{~A}$ & $25,5 \pm 1,9 \mathrm{~A}$ \\
\hline & GSVE & $23,3 \pm 4,0 \mathrm{~A}$ & $24,4 \pm 4,0 \mathrm{~A}$ & $22,8 \pm 2,8 \mathrm{~A}$ & $22,7 \pm 6,0$ & $24,4 \pm 3,1 \mathrm{~A}$ \\
\hline & GIVC & $19,3 \pm 1,6 \mathrm{~B}$ & $18,6 \pm 2,1 \mathrm{~B}$ & $17,7 \pm 1,8 \mathrm{~B}$ & $17,7 \pm 1,8 \mathrm{~B}$ & $17,2 \pm 1,4 \mathrm{~B}$ \\
\hline & GSVC & $18,7 \pm 1,0 \mathrm{~B}$ & $19,9 \pm 2,3 \mathrm{~B}$ & $20,7 \pm 2,3$ & $20,2 \pm 2,5$ & $19,7 \pm 2,2 \mathrm{~B}$ \\
\hline \multirow{4}{*}{ VT exp } & GIVE & $22,3 \pm 2,3 \mathrm{~A}$ & $23,9 \pm 4,2 \mathrm{~A}$ & $21,8 \pm 1,5 \mathrm{~A}$ & $23,8 \pm 2,1 \mathrm{~A}$ & $22,6 \pm 2,5 \mathrm{~A}$ \\
\hline & GSVE & $19,8 \pm 5,2$ & $21,0 \pm 5,7$ & $20,6 \pm 3,0 \mathrm{C}$ & $20,0 \pm 5,7 \mathrm{C}$ & $22,9 \pm 3,2 \mathrm{~A}$ \\
\hline & GIVC & $18,2 \pm 1,0 \mathrm{~B}$ & $17,2 \pm 2,8 \mathrm{~B}$ & $16,7 \pm 2,9 \mathrm{~B}$ & $15,9 \pm 2,9 \mathrm{~B}$ & $15,2 \pm 2,4 \mathrm{~B}$ \\
\hline & GSVC & $16,2 \pm 1,7 \mathrm{~B}$ & $17,5 \pm 2,2 \mathrm{~B}$ & $18,1 \pm 2,2 \mathrm{~B}$ & $17,7 \pm 2,6 \mathrm{~B}$ & $17,4 \pm 1,8 \mathrm{~B}$ \\
\hline \multirow{4}{*}{ VT al } & GIVE & $11,2 \pm 5,2$ & $11,5 \pm 5,4$ & $9,2 \pm 6,5$ & $11,7 \pm 5,4$ & $13,7 \pm 1,2$ \\
\hline & GSVE & $12,3 \pm 2,8$ & $13,0 \pm 2,4 \mathrm{~B}$ & $12,5 \pm 1,6 \mathrm{~B}$ & $13,0 \pm 1,8 \mathrm{~B}$ & $13,7 \pm 2,0$ \\
\hline & GIVC & $8,5 \pm 4,2$ & $5,5 \pm 5,6 \mathrm{~A}$ & $5,7 \pm 5,7 \mathrm{~A}$ & $5,2 \pm 5,2 \mathrm{~A}$ & $4,5 \pm 4,5$ \\
\hline & GSVC & $6,8 \pm 5,0$ & $8,7 \pm 4,5 \mathrm{~A}$ & $8,5 \pm 3,9$ & $7,0 \pm 5,2$ & $8,7 \pm 4,3$ \\
\hline \multirow{4}{*}{ Vd aw } & GIVE & $6,8 \pm 3,2$ & $7,0 \pm 3,3$ & $5,7 \pm 4,0$ & $7,2 \pm 3,2$ & $8,8 \pm 0,9 \mathrm{~B}$ \\
\hline & GSVE & $6,3 \pm 3,0$ & $8,0 \pm 2,2 \mathrm{~B}$ & $7,7 \pm 2,0$ & $8,0 \pm 2,1 \mathrm{~B}$ & $8,3 \pm 1,1 \mathrm{~B}$ \\
\hline & GIVC & $5,7 \pm 2,7$ & $3,3 \pm 3,5 \mathrm{~A}$ & $3,2 \pm 3,3$ & $3,0 \pm 3,1 \mathrm{~A}$ & $3,2 \pm 3,2 \mathrm{~A}$ \\
\hline & GSVC & $7,2 \pm 1,6$ & $7,8 \pm 1,5$ & $7,4 \pm 1,1$ & $7,2 \pm 1,7$ & $7,6 \pm 1,1 \mathrm{~B}$ \\
\hline \multirow{4}{*}{$\mathrm{T}$ insp } & GIVE & $0,4 \pm 0,0 \mathrm{~A}$ & $0,5 \pm 0,1$ & $0,4 \pm 0,1 \mathrm{~B}$ & $0,4 \pm 0,1 \mathrm{~B}$ & $0,4 \pm 0,1 \mathrm{~A}$ \\
\hline & GSVE & $0,6 \pm 0,2 \mathrm{~B}$ & $0,5 \pm 0,2 \mathrm{~A}$ & $0,5 \pm 0,1 \mathrm{~A}$ & $0,6 \pm 0,2 \mathrm{~A}$ & $0,5 \pm 0,1 \mathrm{C}$ \\
\hline & GIVC & $0,4 \pm 0,1$ & $0,4 \pm 0,1 \mathrm{~B}$ & $0,4 \pm 0,1 \mathrm{~B}$ & $0,3 \pm 0,0 \mathrm{~B}$ & $0,3 \pm 0,0 \mathrm{~B}$ \\
\hline & GSVC & $0,3 \pm 0,0$ & $0,3 \pm 0,1$ & $0,3 \pm 0,0 \mathrm{~B}$ & $0,4 \pm 0,0 \mathrm{~B}$ & $0,4 \pm 0,1 \mathrm{~B}$ \\
\hline \multirow{4}{*}{ PEEP } & GIVE & $2 \pm 0$ & $2 \pm 0$ & $2 \pm 1 \mathrm{~A}$ & $2 \pm 1$ & $2 \pm 1$ \\
\hline & GSVE & $2 \pm 1 B$ & $2 \pm 1$ & $2 \pm 0 \mathrm{~A}$ & $2 \pm 0$ & $2 \pm 0$ \\
\hline & GIVC & $2 \pm 0 \mathrm{~A}$ & $2 \pm 0$ & $2 \pm 0$ & $2 \pm 0$ & $2 \pm 0$ \\
\hline & GSVC & $2 \pm 0$ & $2 \pm 0$ & $3 \pm 1 \mathrm{~B}$ & $2 \pm 0$ & $2 \pm 0$ \\
\hline \multirow{4}{*}{ PIP } & GIVE & $2 \pm 1 \mathrm{~A}$ & $2 \pm 1 \mathrm{~A}$ & $2 \pm 0 \mathrm{~A}$ & $2 \pm 1 \mathrm{~A}$ & $1 \pm 0 \mathrm{~A}$ \\
\hline & GSVE & $1 \pm 1 \mathrm{~A}$ & $1 \pm 0 \mathrm{~A}$ & $1 \pm 0 \mathrm{~A}$ & $1 \pm 1 \mathrm{~A}$ & $2 \pm 1 \mathrm{~A}$ \\
\hline & GIVC & $14 \pm 3 B$ & $14 \pm 3 B$ & $14 \pm 2 B$ & $14 \pm 3 \mathrm{~B}$ & $14 \pm 3 B$ \\
\hline & GSVC & $13 \pm 2 \mathrm{~B}$ & $13 \pm 2 \mathrm{~B}$ & $13 \pm 2 B$ & $13 \pm 2 \mathrm{~B}$ & $13 \pm 2 \mathrm{~B}$ \\
\hline \multirow{4}{*}{$\mathrm{PAO} 2$} & GIVE & $576,3 \pm 10,0$ & $579,7 \pm 7,4 \mathrm{~A}$ & $578,5 \pm 9,1 \mathrm{~A}$ & $582,4 \pm 7,9 \mathrm{~A}$ & $583,2 \pm 9,2 \mathrm{~A}$ \\
\hline & GSVE & $541,6 \pm 49,9$ & $541,1 \pm 30,4 \mathrm{~B}$ & $550,1 \pm 35,1 \mathrm{~B}$ & $544,7 \pm 35,2 \mathrm{~B}$ & $526,6 \pm 35,7 \mathrm{~B}$ \\
\hline & GIVC & $570,8 \pm 5,3$ & $568,8 \pm 8,5$ & $563,0 \pm 12,1$ & $563,0 \pm 13,6$ & $554,5 \pm 13,6$ \\
\hline & GSVC & $557,5 \pm 9,8$ & $557,1 \pm 5,0 \mathrm{~A}$ & $554,1 \pm 5,3 \mathrm{~B}$ & $546,8 \pm 7,3 \mathrm{~B}$ & $540,4 \pm 6,6 \mathrm{~B}$ \\
\hline \multirow{4}{*}{$\mathrm{P}(\mathrm{aET}) \mathrm{CO} 2$} & GIVE & $8,3 \pm 7,0$ & $8,8 \pm 6,5$ & $10,3 \pm 6,9 \mathrm{~A}$ & $8,2 \pm 6,4 \mathrm{~A}$ & $8,7 \pm 7,0 \mathrm{~A}$ \\
\hline & GSVE & $20,1 \pm 23,9$ & $27,4 \pm 17,5$ & $28,9 \pm 31,7$ & $26,2 \pm 19,8$ & $36,3 \pm 18,5 \mathrm{~B}$ \\
\hline & GIVC & $14,7 \pm 6,9$ & $21,6 \pm 18,9$ & $17,9 \pm 11,2 \mathrm{~B}$ & $23,2 \pm 11,0 \mathrm{~B}$ & $27,9 \pm 12,7$ \\
\hline & GSVC & $19,3 \pm 7,7$ & $18,1 \pm 7,8$ & $19,4 \pm 7,2$ & $22,2 \pm 7,7$ & $23,8 \pm 9,6$ \\
\hline \multirow{4}{*}{$\mathrm{VM}$} & GIVE & $1,3 \pm 0,3 \mathrm{~A}$ & $1,4 \pm 0,4 \mathrm{~A}$ & $1,5 \pm 0,3 \mathrm{~A}$ & $1,5 \pm 0,3 \mathrm{~A}$ & $1,4 \pm 0,4 \mathrm{~A}$ \\
\hline & GSVE & $0,8 \pm 0,3 \mathrm{~B}$ & $1,0 \pm 0,4$ & $0,9 \pm 0,3 \mathrm{~B}$ & $0,9 \pm 0,3 \mathrm{~B}$ & $1,0 \pm 0,4$ \\
\hline & GIVC & $1,0 \pm 0,2$ & $0,9 \pm 0,2 \mathrm{~B}$ & $0,9 \pm 0,2 \mathrm{~B}$ & $0,8 \pm 0,2 \mathrm{~B}$ & $0,8 \pm 0,2 \mathrm{~B}$ \\
\hline & GSVC & $1,0 \pm 0,2$ & $1,0 \pm 0,2$ & $1,0 \pm 0,1 \mathrm{~B}$ & $1,0 \pm 0,2 \mathrm{~B}$ & $1,0 \pm 0,2$ \\
\hline \multirow{4}{*}{ Vd alv } & GIVE & $9,0 \pm 4,2$ & $7,3 \pm 5,2$ & $5,3 \pm 5,3$ & $7,2 \pm 5,1$ & $7,2 \pm 5,1$ \\
\hline & GSVE & $11,2 \pm 1,2$ & $11,0 \pm 1,3 \mathrm{~A}$ & $10,8 \pm 1,1 \mathrm{~A}$ & $10,7 \pm 1,2 \mathrm{~A}$ & $11,2 \pm 1,3$ \\
\hline & GIVC & $8,3 \pm 3,9$ & $5,0 \pm 5,0 \mathrm{~B}$ & $4,8 \pm 4,9 \mathrm{~B}$ & $4,8 \pm 4,9 \mathrm{~B}$ & $5,7 \pm 6,3$ \\
\hline & GSVC & $7,2 \pm 3,4$ & $5,0 \pm 4,0 \mathrm{~B}$ & $7,8 \pm 1,6$ & $8,2 \pm 1,7$ & $8,3 \pm 1,5$ \\
\hline
\end{tabular}

Médias seguidas por letras maiúsculas diferentes, na coluna, diferem entre si pela análise de perfil $(\mathrm{P}<0,05)$. Médias seguidas por letras minúsculas diferentes, na linha, diferem entre si pela análise de perfil $(\mathrm{P}<0,05)$. SpO2: saturação de oxi-hemoglobina por oximetria de pulso; ETCO2: tensão de dióxido de carbono ao final da expiração; FR: frequência respiratória; VT ins e exp: volumes correntes inspiratório e expiratório; VT al: volume corrente alveolar; Vd aw: espaço morto das vias aéreas; T insp: tempo inspiratório; PEEP: pressão positiva ao final da expiração; PIP: pico de pressão inspiratória; PAO2: pressão parcial alveolar de oxigênio; $\mathrm{P}(\mathrm{a}-\mathrm{ET}) \mathrm{CO}_{2:}$ diferença de tensão de dióxido de carbono; VM: volume minuto; Vd alv: espaço morto alveolar.

M0: mensuração 60 minutos após a indução; M15: mensuração 15 minutos após o bolus de rocurônio (GIVC, GSVC) ou NaCl 0,9\% (GIVE, GSVE); M30: mensuração 30 minutos após o bolus de rocurônio (GIVC, GSVC) ou NaCl 0,9\% (GIVE, GSVE); M45: mensuração 45 minutos após o bolus de rocurônio (GIVC, GSVC) ou NaCl 0,9\% (GIVE, GSVE); M60: mensuração 60 minutos após o bolus de rocurônio (GIVC, GSVC) ou NaCl 0,9\% (GIVE, GSVE). 


\section{CONCLUSÕES}

A ventilação controlada a volume não é capaz de manter a normocapnia pretendida em coelhos, gerando-lhes acidose, principalmente quando se utiliza o sevofluorano como agente anestésico. $\mathrm{O}$ emprego da ventilação controlada a volume, utilizando isofluorano como anestésico em coelhos, não proporciona maior oxigenação alveolar em relação à ventilação espontânea. O uso do isofluorano em coelhos demonstra maior estabilidade anestésica que o sevofluorano, pela análise das características avaliadas.

\section{REFERÊNCIAS}

AMARAL, J.L.G.; FERREIRA, A.C.P.; FEREZ, D.; GERETTO, P. Monitorização da respiração: oximetria e capnografia. Rev. Bras. Anestesiol., v.42, p.51-58, 1992.

BARZAGO, M.M.; BORTOLOTTI, A.; OMARINI, D. et al. Monitoring of blood gas parameters and acid-base balance of pregnant and non-pregnant rabbits (Oryctolagus cuniculus) in routine experimental conditions. Lab. Anim., v.26, p.73-79, 1992.

BATISTA, P.A.C.S. Estudo comparativo entre as ventilações espontânea, mandatória intermitente sincronizada, pressão de suporte $e$ volume garantido e suporte pressórico, em coelhos, anestesiados com propofol e induzidos à hipovolemia aguda. 2011. 149f. Tese (Doutorado em cirurgia veterinária) - Faculdade de Medicina Veterinária e Zootecnia, Universidade Estadual Paulista - São Paulo.

BONETTI, E.; DALLAN, L.A.O. Hemodinâmica básica e avançada. In: FELIX, V.N. et al. Terapia intensiva - adulto - pediatria/RN. São Paulo: Sarvier, 1997. p. 40-45.

BORGES, P.A.; NUNES, N.; CAMACHO, A.A. et al. Diferentes frações inspiradas de oxigênio em coelhos hipovolêmicos anestesiados com propofol e submetidos à ventilação mecânica. Cienc. Rur., v.41, p.1960-1966, 2011.

CURI, P.R. Análise de medidas repetidas em experimentos biológicos. Rev. Bras. Estat., v.41, p.137-150, 1980.

DAVID, C.M. Ventilação mecânica repercussões hemodinâmicas. In: DAVID, C.M. Ventilação mecânica. Rio de Janeiro: REVINTER, 2011. Cap. 22, p.215-224.
FERNANDES, C.R.; RUIZ NETO, P.P. O sistema respiratório e o idoso: implicações anestésicas. Rev. Bras. Anestesiol., v.52, p.461470, 2002.

FLECKNELL, P.A.; ROUGHAN, J.V.; HEDENQVIST, P. Induction of anaesthesia with sevoflurane and isoflurane in the rabbit. $L a b$. Anim., v.33, p.41-46, 1999.

FONSECA, E.B. Comparação da variação da pressão sistólica e de pulso nas ventilações com pressão e volume controlados: estudo experimental em coelhos. 2006. 140f. Tese (Doutorado em Ciências) - Faculdade de Medicina da Universidade de São Paulo, São Paulo.

FONSECA, N.M.; GOLDENBERG, S.; GOMES, P.O.; LIMA, A.P. Anestesia em coelho. Acta. Cir. Bras., v.11, p.82-104, 1996.

GALAS, F.R.B.G.; ALMEIDA, J.P.; AULER Jr., J.O.C. Variáveis da monitorização da ventilação mecânica intraoperatória. In: POTÉRIO, G.M.B.; PIRES, O.C.; CALLEGARI, D.C.; SLULLITEL, A. Monitorização em anesthesia. São Paulo: MANOLE, 2011. p.1-7.

JERRE, G.; SILVA, T.J.; BERALDO, M.A. et al. Fisioterapia no paciente sob ventilação mecânica. J. Bras. Pneumol., v.33, p.142-150, 2007.

JOHSON, R.A.; MORAIS, H.A. Distúrbios ácido-básicos respiratórios. In: DiBARTOLA, S.P. Anormalidades de fluidos, eletrólitos $e$ equilíbrio ácido-básico na clínica de pequenos animais. São Paulo: ROCA, 2007. p.270-283.

LIMA, C.A.P.; GOLDENBERG, S.; FONSECA, N.M. et al. Avaliação de um sistema circular de anestesia, em ventilação mecânica com pressão positiva intermitente. Anestesia quantitativa em coelhos (Oryctolaguscuniculus). Acta. Cir. Bras., v.11, p.108-115, 1996.

MARTINS, S.E.C.; NUNES, N.; REZENDE, M.L.; SANTOS, P.S.P. Efeitos do desfluorano, sevofluorano e isofluorano sobre variáveis respiratórias e hemogasométricas em cães. Bras. J. Vet. Res. Anim. Sci., v.40, p.190-196. 2003.

MORRISON, D.F. Multivariate statistical methods. In: MAC GROWS. New York: HILL BOOK CO, 1967. 388p. 
ROTTA, A.T.; STEINHORN, D.M. Ventilação mecânica convencional em pediatria. J. Pediatr., v.33, p.100-108, 2007.

TOUFEN JUNIOR, C.; CARVALHO, C.R.R. Ventiladores mecânicos. J. Bras. Pneumol., v.33, p.71-91, 2007.

VALE, N.B. Princípios de farmacodinâmica de drogas anestésicas. Rev. Bras. Anestesiol., v.44, p.13-23, 1994.
WEISMAN, I.M.; RINALD，J.E.; ROGERS, R.M. et al. State of the art: intermittent mandatory ventilation. Am. Rev. Respir. Dis., v.127, p.641, 1983.

WEST, J.B. Relações de ventilação - perfusão como o equilíbrio entre o sangue e o gás determina a troca gasosa. In: WEST, J.B. Fisiologia Respiratória. São Paulo: MANOLE, 2002. p.31-51. 